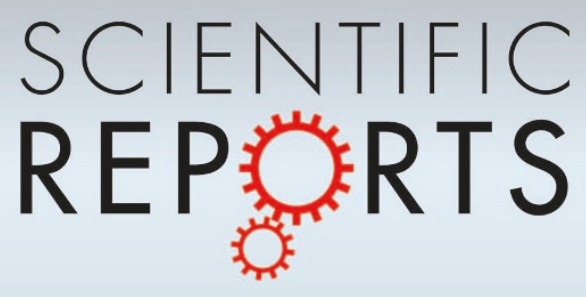

\title{
SUBJECT AREAS: \\ COMPUTATIONAL \\ CHEMISTRY \\ Regulating the Transport of DNA through Biofriendly Nanochannels in a Thin Solid Membrane
}

BIOMEDICAL ENGINEERING

NANOPORES

COMPUTATIONAL BIOPHYSICS

Received

21 May 2013

Accepted

20 January 2014

Published

5 February 2014

Correspondence and requests for materials should be addressed to B.Q.L. (bluan@us.ibm. com) or G.S.

(Gustavo@us.ibm. com)

* These authors contributed equally to this work.

\author{
Deqiang Wang ${ }^{1 *}$, Stefan Harrer ${ }^{2 *}$, Binquan Luan ${ }^{*}$, Gustavo Stolovitzky' ${ }^{1}$, Hongbo Peng ${ }^{1}$ \\ \& Ali Afzali-Ardakani'
}

'IBM T J Watson Research Center, 1101 Kitchawan Road, Yorktown Heights, NY, 10598, USA, ²IBM Research Australia, 204 Lygon Street, 3053 Carlton, VIC, Australia.

Channels formed by membrane proteins regulate the transport of water, ions or nutrients that are essential to cells' metabolism. Recent advances in nanotechnology allow us to fabricate solid-state nanopores for transporting and analyzing biomolecules. However, uncontrollable surface properties of a fabricated nanopore cause irregular transport of biomolecules, limiting potential biomimetic applications. Here we show that a nanopore functionalized with a self-assembled monolayer (SAM) can potentially regulate the transport of a DNA molecule by changing functional groups of the SAM. We found that an enhanced interaction between DNA and a SAM-coated nanopore can slow down the translocation speed of DNA molecules and increase the DNA capture-rate. Our results demonstrate that the transport of DNA molecules inside nanopores could be modulated by coating a SAM on the pore surface. Our method to control the DNA motion inside a nanopore may find its applications in nanopore-based DNA sequencing devices.

rotein channels, embedded in a cell membrane, connect the cytoplasm of a cell with an extracellular fluid, playing an important role in transporting molecules in and out of a cell. Functional membrane channels include aquaporins that enhance the membrane permeability of water ${ }^{1}$, ion channels that selectively transport protons, potassium, sodium or calcium ions ${ }^{2}$, active transporters that bring in small nutrients such as vitamin $B_{12}$, and so on. The high selectivity of each type of membrane channels, resulting from subtle and energetically favorable interactions between a transported solute and the channel's surface, ensures that other solutes are excluded. Inspired by transport mechanisms of membrane proteins, researchers have designed and built biomimetic channels or nanopores in a thin solid membrane. As a single-molecule technique, biomemetic channels can not only be used to transport ions or molecules but also be deployed to analyze transported solutes ${ }^{3-5}$. Due to its confined geometry, comparable to the size of a transported solute, a synthetic nanopore has been widely used as a sensor for structural analyses of a transported molecule, such as a DNA molecule with bound proteins ${ }^{6,7}$.

However, because of the dynamic drilling process by a focused ion beam ${ }^{8}$ or a transmission electron ${ }^{3}$ beam, $^{2}$ each solid-state nanopore typically has its unique and uncontrolled surface features (e.g. charge distribution). Experimentally, it has been shown that interaction between DNA and the surface of a solid-state nanopore can affect the transport dynamics of the DNA ${ }^{9}$. It is therefore essential to improve surface properties and avoid intermittent events of DNA sticking to the surface. Several methods have been developed, including assembling a fluidic lipid-bilayer on the surface of a solid-state nanopore ${ }^{10}$, coating DNA hairpins to the pore surface ${ }^{11}$, embedding a protein nanopore inside the solid-state one ${ }^{12}$, inserting a DNA origami pore inside the solid-state one $^{13}$, chemically modifying the pore surface with organic molecules ${ }^{14-16}$, etc. Closely related to the last method, we suggest to coat a self-assembled monolayer (SAM) on the pore surface and modify functional groups (via chemical reaction) on the SAM surface to achieve improved transport of DNA.

By flushing chemical agents through a solid-state nanopore with a SAM containing 4-carboxyl benzyl phosphonic acids, we were able to in - situ switch the functionality of the SAM from being hydrophilic to being hydrophobic. Such change results in a reduced interaction between DNA and the coated pore. Theoretically, increasing the DNA-pore interaction can slow down the DNA translocation speed and increase the capture-rate at the same time. An optimum transport flux can be achieved for the intermediate-interaction region ${ }^{17}$. In this paper, we show that DNA interacts more strongly with the hydrophilic-SAM-coated pore than it does with the hydrophobic-SAM-coated one. Consistent with theoretical results, we found that in the hydrophilic-SAM-coated 
pore the DNA's translocation speed is slower but the capture rate is actually larger than in the hydrophobic-SAM-coated one. Our work highlights the possibility to fine-tune surface properties of a nanopore and to achieve desired transport rates of DNA molecules.

\section{Results}

Experiment of DNA translocation through SAM-coated nanopores. Figure 1a illustrates our experimental set-up. Two fluidic chambers (cis. and trans.) are separated by a $30-\mathrm{nm}$ thick $\mathrm{Si}_{3} \mathrm{~N}_{4}$ membrane and are connected by a nanopore drilled into the membrane. 4-carboxyl benzyl phosphonic acid molecules were used to coat the nanopore surface and the membrane surface. Figure $1 \mathrm{~b}$ and Figure $1 \mathrm{c}$ respectively show TEM images of a nanopore before and after the coating. After the coating, a self-assembled monolayer (SAM) covers the surface of a solid-state nanopore and the radius of the pore is reduced. For each molecule in the SAM, the end containing a phosphonic acid group can covalently self-assemble to the pore surface. By means of organic syntheses on the other end of a coated molecule, different chemical groups can be positioned on the SAM surface, resulting in different functionalities of the SAM-coated nanopore. Here, we used trimethylsilyl diazomethane, as a methylating agent, to methylate carboxyl groups on the hydrophilic SAM surface, switching the hydrophilic SAM surface into the hydrophobic one (Figure 1d). Because of the solubility and the size of molecules in a desired SAM, we found that it was efficient to coat 4-carboxyl benzyl phosphonic acid molecules on the pore surface first and then to switch functional groups to target ones by esterification of carboxylic acid.

To study the transport of biomolecules in differently functionalized nanopores, we loaded DNA molecules $(20-\mathrm{nM})$ in a $1-\mathrm{M} \mathrm{KCl}$ electrolyte in the cis. chamber and applied a biasing electric field to drive DNA molecules through functionalized nanopores (Figure 1a). With the biasing electric field, an ionic current $i_{\text {ion }}$ through the pore can be measured. During the DNA translocation, such current is reduced due to the physical blockage by a DNA molecule ( $\Delta I$ shown in Figure 1a). The duration of current blockage, or the residence time of DNA, can be used to calculate the translocation speed of DNA. Thus, when DNA transits a functionalized nanopore, the complex interplay of electric, hydrodynamic and tribological interactions on a

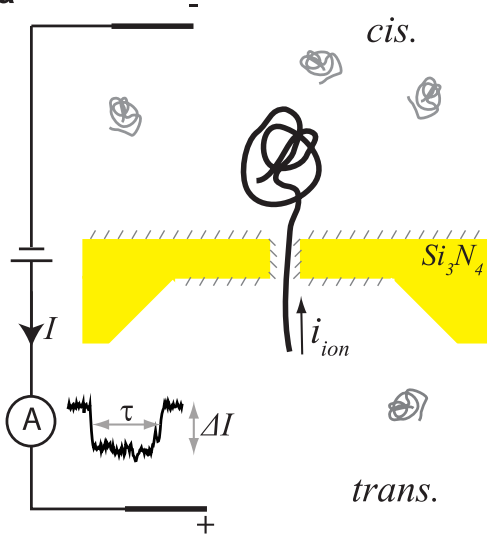

b

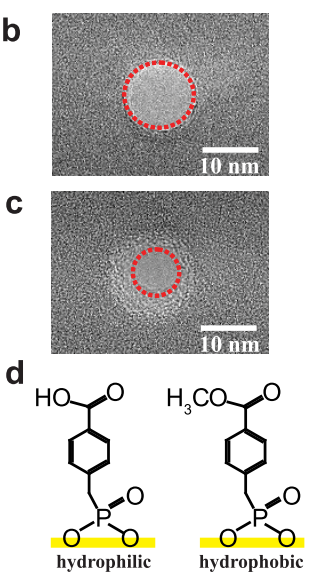

Figure 1 Experimental set-up. (a) A schematic of DNA translocation (driven by a biasing electric field) through a SAM-coated nanopore. (b), (c) TEM images of a solid-state nanopore (12-nm-in-diameter) before and after coating with carboxyl benzyl phosphonic acid, respectively. Red circles highlight pore sizes. (d) Configurations of molecules in the hydrophilic and hydrophobic SAMs. The carboxyl benzyl phosphonic acid was used to coat a solid-state nanopore in a 30-nm-thick $S i_{3} N_{4}$ membrane. The other end can be changed from hydrophilic to hydrophobic status with trimethylsilyl diazomethane.
DNA could be revealed from fingerprints (such as $\tau$ and $\Delta I$ ) of an ion-current trace.

Because of the drilling process of nanopores, each pore has its unique physical and geometrical properties that affect the ion conductivity. To pinpoint coating effects by different SAMs, we report experimental results obtained for the same pore (about $5 \mathrm{~nm}$ in diameter and about $4 \mathrm{~nm}$ in diameter after the coating). Similar experiments were repeated for different pores and results were consistent (supporting information). We observed DNA translocation events before the pore was fully wetted. Therefore, we only analyzed current data after the open-pore current reached its maximum value. Figure 2a and Figure $2 \mathrm{~b}$ show 4 seconds of typical ion-current traces for DNA translocations through the same pore with hydrophilicSAM-coated and hydrophobic-SAM coated surfaces, respectively. In each case, 2 -Kbp DNA molecules were electrophoretically driven through the pore at a biasing voltage of $300 \mathrm{mV}$. Averaged over translocation events (number $N$ ), the dwell time of DNA in the hydrophilic-SAM-coated nanopore is about $0.4 \mathrm{~ms}(\mathrm{~N}=1451$, Figure 2c), about 2.7 times longer than the mean dwell time of $0.15 \mathrm{~ms}$ for DNA in the hydrophobic- SAM-coated pore $(\mathrm{N}=$ 1942 , Figure 2d). This indicates that the pore surface is lubricated by the hydrophobic SAM. Because of reduced interaction (e.g. sticking) between DNA and the pore, DNA translocation traces tended to last longer for the hydrophobic-SAM-coated nanopore. The longest recorded trace of free DNA translocations corresponded to the pore coated with the hydrophobic SAM and lasted $160 \mathrm{~s}$ (Supplementary Information).

From measured open-pore currents (Fig. 2a and Fig. 2b), the average open-pore conductances were estimated to be $12.2 \pm$ $0.5 \mathrm{nS}$ for the hydrophilic pore and $14.5 \pm 1 \mathrm{nS}$ for the hydrophobic pore, which are of the right order of magnitude (6 nS) calculated ${ }^{18}$ for the geometry of the pore considered. In a hydrophilic-SAM-coated nanopore, the SAM surface serves as a nonslip boundary for the confined electrolyte and velocities of ions and water approach zero near the surface. Therefore, the larger open-pore current in a hydrophobic-SAM-coated nanopore is due to finite mobilities of ions near such surface (serving as a slipboundary).

The capture rates (i.e. the number of translocation events per unit time) are $38.4 \mathrm{~Hz}$ and $24.3 \mathrm{~Hz}$ for DNA transiting a hydrophilicSAM-coated and a hydrophobic-SAM-coated pore (Figure 2a and
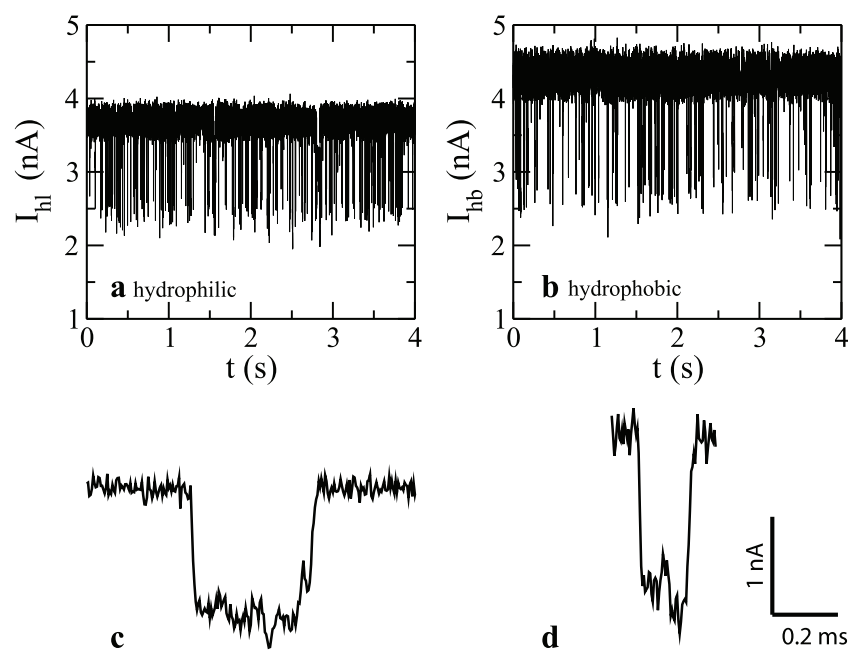

Figure $2 \mid$ Translocation of DNA through the same nanopore coated with different SAMs at $300 \mathrm{mV}$. (a) hydrophilic-SAM coating; (b) hydrophobic-SAM coating, switched from the hydrophilic-SAM coating after adding trimethylsilyl diazomethane. (c), (d) typical electric signals of DNA translocation events. 
Figure 2b), respectively. It is worth noting that the event frequency of the hydrophobic-SAM-coated nanopore is $63 \%$ that of the hydrophilic-SAM-coated one. This indicates that the probability of capturing DNA by the hydrophilic-SAM-coated nanopore is larger than that by the hydrophobic-SAM-coated one. Theoretically, this can be accounted for ${ }^{17}$ by enhanced interaction between DNA and the pore surface (demonstrated below). Event frequencies at higher biasing voltages are summarized in Table 1, showing consistently higher frequencies in a hydrophilic-SAM-coated nanopore. The capture rate increased when the biasing voltage ${ }^{19}$ changed from $300 \mathrm{mV}$ to $400 \mathrm{mV}$. At an even higher biasing voltage $(500 \mathrm{mV})$, the capture rate was reduced likely because of the stronger interaction (in a higher electric field) between the DNA and the SAM on the top surface of the membrane (Fig. 1a).

Figure 3 a shows the scatter plots of conductance changes versus dwell times for both cases. Two well-defined clusters can be identified, corresponding to two distinctive dynamics of DNA transport in two-SAM-coated nanopores. Besides different residence times of DNA in SAM-coated pores, translocation-induced current blockades are also different. At a biasing voltage of $300 \mathrm{mV}$, average blockade currents, with standard deviations of the mean, were $1,200 \pm 28 \mathrm{pA}$ and $1,529 \pm 37 \mathrm{pA}$ for hydrophilic-SAM-coated and hydrophobic-SAM-coated pores, respectively.

The distributions of blockade currents, shown in Figure $3 \mathrm{~b}$ are Gaussian-like ${ }^{3}$, similar to previous experimental results for DNA translocation in a bare solid-state nanopore. The wider distribution of the conductance change for the hydrophobic pore may result from larger fluctuation at the water-SAM (hydrophobic) interface.

For comparison, we normalized the mean current blockages with their respective open-pore currents. The normalized values are $32.4 \%$ and $35.6 \%$ for hydrophilic-SAM-coated and hydrophobicSAM-coated nanopores. Note that the normalized value for the hydrophobic-SAM-coated nanopore is about $10 \%$ larger than that for the hydrophilic-SAM-coated one. Theoretically, for a high-ionconcentration electrolyte (such as $1 \mathrm{M}$ used in our experiment), normalized blockade currents can be simply estimated by $S_{\text {DNA }} /$ $S_{\text {pore }}$, where $S_{\text {DNA }}$ and $S_{\text {pore }}$ are the cross-section areas of DNA and the coated pore. As shown in the supporting materials, because of the hydrophobic-SAM surface, the effective radius of the pore (accessible by water) is $1.9 \mathrm{~nm}$ instead of $2 \mathrm{~nm}$ for the hydrophilic-SAM-coated one. Thus, the resulting cross-section area for the hydrophobicSAM-coated nanopore is about $10 \%$ lesss than that for the hydrophilic-SAM-coated one, which accounts for the difference between normalized values of current blockades for two SAM-coated pores. For the hydrophilic-SAM-coated pore, if the radius of DNA is $1.1 \mathrm{~nm}$, the predicted normalized value is $30.3 \%$, consistent with the experimentally obtained value.

Figure $3 \mathrm{c}$ and Figure $3 \mathrm{~d}$ show the trends regarding the average dwell time and the average conductance change, as a function of biasing voltages, for hydrophilic-SAM-coated and hydrophobicSAM-coated nanopores. The dwell time decreases as the voltage increases from $300 \mathrm{mV}$ to $500 \mathrm{mV}$ for both types of pores. The translocation of $2 \mathrm{Kbp}$ DNA in the hydrophilic-SAM-coated nanopore is at least twice slower than in the hydrophobic-SAM-coated one, with biasing voltages ranging from $300 \mathrm{mV}$ to $500 \mathrm{mV}$. Most events with long dwell times in Figure 3a (e.g. from 2 ms to $100 \mathrm{~ms}$ ) correspond to translocation of DNA through the hydrophilic-SAM-

Table 1 | Frequencies $(\mathrm{Hz}) \mathrm{f}$ of DNA translocation events in two SAM-coated nanopores at different biasing voltages $(\mathrm{mV})$

\begin{tabular}{lccc} 
& $300 \mathrm{mV}$ & $400 \mathrm{mV}$ & $500 \mathrm{mV}$ \\
\hline hydrophobic-SAM $f_{1}$ & 24.3 & 39.6 & 31.2 \\
hydrophilic-SAM $f_{2}$ & 38.4 & 56.9 & 53.9 \\
ratio $f_{1} / f_{2}$ & $63 \%$ & $70 \%$ & $58 \%$ \\
\hline
\end{tabular}
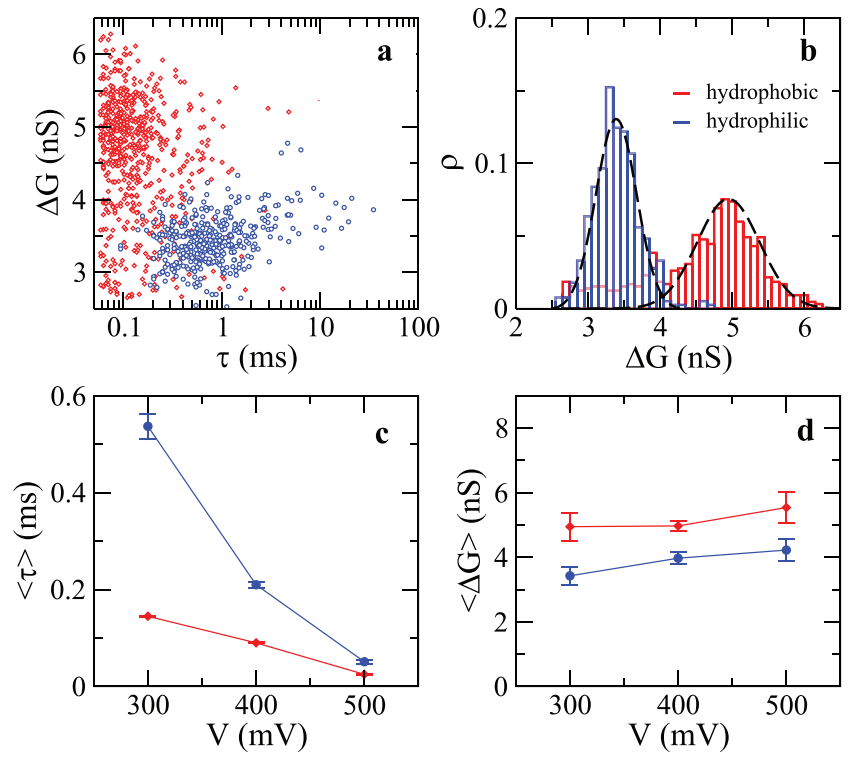

Figure $3 \mid$ Comparison of DNA translocations through the same nanopore coated with hydrophilic- and hydrophobic-SAMs. (a) The scatter plots of conductance changes versus dwell times for both SAMcoatings. $V=300 \mathrm{mV}$. Numbers of events are 659 and 395 for hydrophobic- and hydrophilic- SAM-coated pores, respectively. (b) The distributions of conductance changes for both SAM-coatings. $V=$ $300 \mathrm{mV}$. The dashed curves in (b) are Gaussian fits to the distributions. (c) Dwell times versus biasing voltages. (d) Conductance changes versus biasing voltages.

coated pore, likely due to enhanced interaction between DNA and the SAM. The blockade current increases in an approximately linear fashion for both cases when the voltage increases and, correspondingly, the conductance changes are nearly constant (Figure 3d). Conductance changes measured for the hydrophilic-SAM-coated pore are about $80 \%$ of those for the hydrophobic-SAM-coated one. Figure S1 in the Supplementary Information shows 1.5 seconds of typical ion-current traces at 300,400 , and $500 \mathrm{mV}$ for DNA translocations in the pores with hydrophilic and hydrophobic SAMs.

Note that we repeated the same experiment for different nanopores with the same SAM coating. We found that results are very consistent. Details are presented in the Supplementary Information (section 6: Fig. S5-S8 and Table AT1).

Molecular dynamics simulation. To interpret experimental results, we carried out all-atom MD simulations of DNA transiting a functionalized nanopore on the IBM Bluegene supercomputer. Figure $4 \mathrm{a}$ illustrates the simulation system that mimics the experimental set-up. The 6-nm-diameter channel was made from the amorphous $\mathrm{SiO}_{2}$ solid. Octanol or octane molecules were covalently linked to $\mathrm{Si}$ atoms that were evenly distributed on the channel surface ${ }^{20}$, which respectively results in a hydrophilic or hydrophobic SAM on the channel surface. Note that, limited by the availability of force fields, the composition of a simulated SAM is different from that in experiment. However, both the pore radii after the coating and functional chemical groups on a SAM-coated surface, are the same in simulation and in experiment. A dsDNA molecule was solvated in a $100 \mathrm{mM} \mathrm{KCl}$ electrolyte. The complex of the DNA and the electrolyte was confined inside the functionalized nanopore. Periodic boundary conditions were applied in all three directions in simulation. The length of the dsDNA molecule is the same as the channel length. End effects (such as slow entry into and fast exit away from a nanopore) of dsDNA were not present because of the covalent linking of the $5^{\prime}$-end and the 3 'end (in each DNA strand) across the periodic boundary. A biasing electric field, 

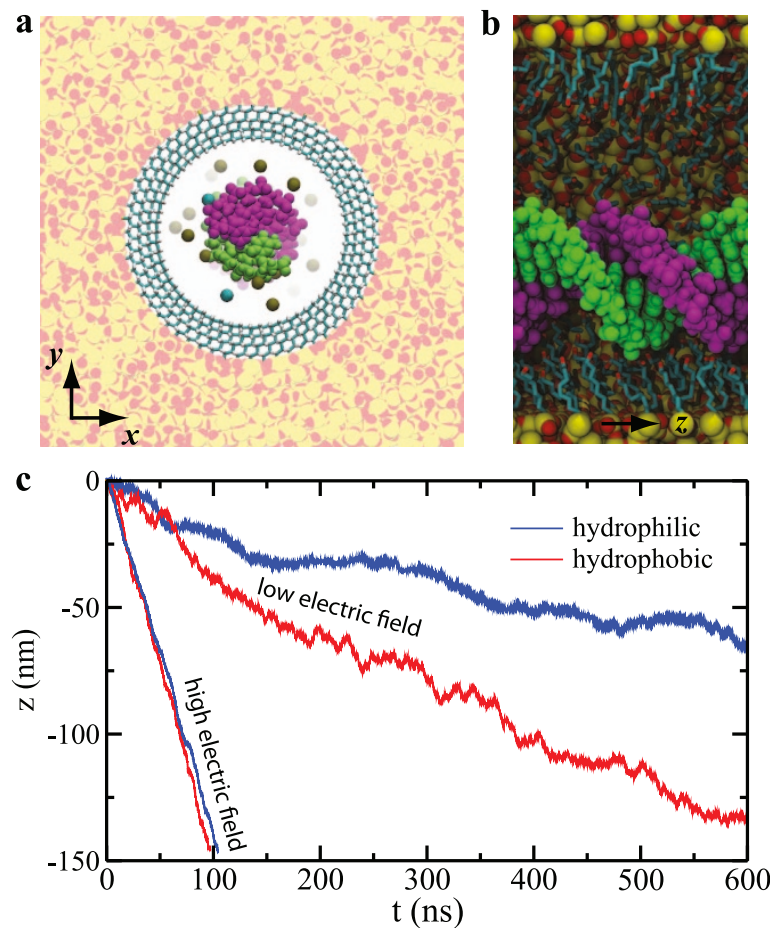

Figure $4 \mid$ MD simulation of DNA transiting a nanopore coated with a hydrophilic- or hydrophobic-SAM. (a) Top view of the simulation system for DNA in a hydrophobic-SAM-coated nanopore. Atoms in the amorphous $\mathrm{SiO}_{2}$ solid are shown as yellow (Si) and red (O) spheres. Molecules in the SAM are in the "stick" representation. Two strands in the dsDNA molecule are colored in purple and green. Potassium and Chlorine ions are shown as tan and cyan balls, respectively. Water molecules are not shown. (b) Side view of the simulation system for DNA in a hydrophilicSAM-coated nanopore. (c) Longitudinal motion (along the $z$ axis) of DNA in SAM-coated nanopores, driven by a low $(3.1 \mathrm{mV} / \mathrm{nm})$ and a high $(31 \mathrm{mV} / \mathrm{nm})$ electric fields.

$3.1 \mathrm{mV} / \mathrm{nm}$ (about $100 \mathrm{mV}$ biasing in experiment), was applied in the $z$ direction to drive the dsDNA molecule through the channel. Details on the simulation set-up are provided in the "method" section.

The translocation of DNA in each SAM-coated nanopore was simulated for $600 \mathrm{~ns}$. Figure 4c shows different trajectories of DNA opposite the electric field direction in two SAM-coated nanopores. In a hydrophobic-SAM-coated nanopore, the mean translocation velocity of DNA is about $0.21 \mathrm{~nm} / \mathrm{ns}$. At short time scales (e.g. $10 \mathrm{~ns}$ ), the velocity of DNA fluctuates and even the reversal motion (in the direction of the electric field) of DNA was observed. In the weak electric field applied in simulation, it is possible that in a short time interval the electroosmotic flow near the DNA surface exerts a larger hydrodynamic friction force than the electric driving force. When DNA is in the hydrophilic-SAM-coated nanopore, the motion of DNA adopts a stick-slip fashion. It is conceivable that DNA can have a stronger interaction with a hydrophilic SAM than with a hydrophobic SAM (see below). Generally, when DNA is contacting the SAM (e.g. around 200 ns in Figure 4c), DNA either stops or moves slower (than near the channel center). The mean velocity of DNA in the hydrophilic nanopore is $0.11 \mathrm{~nm} / \mathrm{ns}$, about $50 \%$ of the translocation velocity of DNA in a hydrophobic nanopore. Experimentally, the mean translocation velocity can be estimated by $L / \tau$, where $L$ is the contour length of the $2 \mathrm{Kbp}$ dsDNA. As shown in Figure 3, percentages derived from experimental results range from $30 \%$ to $50 \%$. Therefore, simulation and experimental results are comparable. The simulated transport velocities of DNA are higher than those obtained in experiment because (1) the diffusion coefficient of a modeled water molecule (TIP3P) is three times higher than that of a real water molecule and (2) the ion concentration $(0.1 \mathrm{M})$ in simulation is 10 times less than that in experiment, resulting in less screening of the DNA charge (or a stronger electric driving force $)^{21}$. Including all these effects, calculated dwell times from simulations are about two to three times less than experimentally measured ones when using the equation $\tau \sim L D \xi /\left(q_{\mathrm{eff}} V\right)$. Here, $\xi$ is the friction coefficient; $D$ is the length of the pore and $q_{\text {eff }}$ is the effective charge of the DNA molecule. More comparisons between simulation and experimental results are shown in the Supplementary Information.

Note that the reversal motion of DNA in the hydrophilic-SAM coated nanopore rarely occurs. Because the hydrophilic SAM serves as a no-slip boundary, part of the momentum of the electroosmotic flow, between the DNA and the SAM surfaces, is transferred to the SAM surface. The friction force on DNA resulting from the electroosmotic flow in a hydrophilic-SAM coated channel is therefore less than that for DNA in a hydrophobic-SAM coated channel.

Figure 5a shows the time-dependent radial motion of DNA during the translocation (also see movies in Supplementary Information). When DNA was in a hydrophobic-SAM-coated nanopore, DNA was always near the center of the channel and was at most a few angstroms away from the center. From an energy point of view, the solvation-energy barrier prevents the hydrophilic DNA molecule from contacting a hydrophobic SAM. Thus, the electric driving force is balanced only by the hydrodynamic friction. When DNA was in a hydrophilic-SAM-coated nanopore, DNA can move away from the center and temporarily stay at the SAM surface. The force resisting DNA's driven motion includes both the hydrodynamic friction and the contact friction between DNA and the SAM. Therefore, as shown in Figure 4c, DNA transits faster in the hydrophobic-SAM-coated pore than in the hydrophilic-SAM-coated one.

Figure $5 \mathrm{~b}$ and $5 \mathrm{c}$ show density functions, $\rho(r)$, illustrating radial distributions of DNA in hydrophilic-SAM-coated and hydrophobicSAM-coated pores. The probability to find DNA, at a distance of $r$ measured from the central axis of DNA to the central axis of a coated

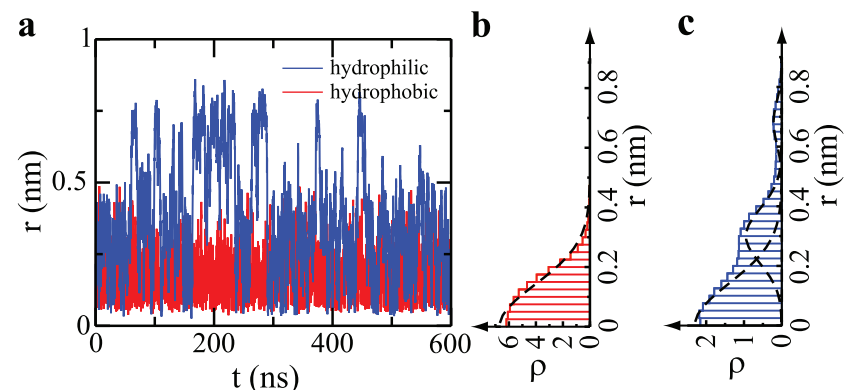

d
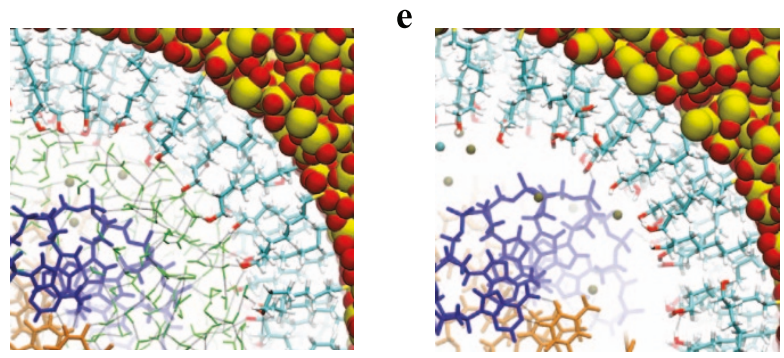

Figure $5 \mid$ Radial motion of DNA in SAM-coated nanopores. a) Timedependent positions of DNA, in the radial direction. (b,c) Radial distribution of DNA in a hydrophobic-SAM-coated (b) and a hydrophilicSAM-coated (c) nanopore. Dashed lines show Gaussian distributions. (d) Illustration of water-mediated interaction between DNA and a hydrophilic SAM. Water molecules are shown as green sticks.

(e) Illustration of the direct interaction between DNA and a hydrophilic SAM. In (d) and (e), DNA is at the lower left corner; part of the $\mathrm{SiO}_{2}$ solid surface is at the upper right corner; the hydrophilic SAM is in the "stick" representation. Hydrogen bonds are presented as gray lines. 
pore, is $\rho(r) 2 \pi r \cdot d r$. When DNA is in the hydrophobic-SAM-coated nanopore, the radius-dependent density function satisfies a singleGaussian distribution centered at the origin (Figure 5b). Thus, DNA is effectively confined near the center of a pore.

When DNA is in the hydrophilic-SAM-coated nanopore, DNA can diffuse away from the pore center and temporarily stay at the coated surface. Intuitively, two Gaussian peaks should be present in the density function, representing higher probability-densities at the center and at the pore surface. Surprisingly, between those two peaks mentioned above, there is an extra Gaussian peak, centered at a distance of $0.36 \mathrm{~nm}$ from the pore center. From simulation trajectories when DNA motion stalls, we found that DNA could interact with the hydrophilic SAM in two different ways. Figure $5 \mathrm{~d}$ shows that the interaction between DNA and the hydrophilic SAM is mediated by a thin (3-4 $\AA$ ) layer of water molecules. When confined between two hydrophilic surfaces close to each other, water molecules exhibit dramatically slow dynamics because of hydrogen bonding to both surfaces (i.e. surface-induced solidification of water). Such watermediated adhesion between DNA and the SAM surface could temporarily stall DNA motion. Another type of interaction is that DNA contacts the hydrophilic SAM-surface directly. As shown in Figure 5e, phosphate groups of DNA form hydrogen bonds with hydroxyl groups on the SAM surface, interlocking two surfaces. Because the radius of DNA is about $1.2 \mathrm{~nm}$ and the radius of a SAM-coated nanopore is about $2 \mathrm{~nm}$, DNA is about $0.7 \mathrm{~nm}$ away from the pore center when the contact (defined when the distance between two surfaces is about $0.1 \mathrm{~nm}$ ) occurs. This is consistent with the Gaussian peak shown at $r=0.7 \mathrm{~nm}$ in Figure 5c.

The above two mechanisms of interfacial locking rely on the formation of hydrogen bonds (between two surfaces) that normally require a typical time $t$. If DNA moves fast, $\sigma / v<t$, where $\sigma$ is a typical shearing distance of one surface relative to the other one before the interfacial locking occurs. Hydrogen bonds between DNA and the SAM-coated surface are always disrupted by the fast shearing process. Therefore DNA cannot be stalled if the translocation velocity $v$ of DNA is too fast. This is confirmed in an independent set of simulations when the electric field strength is 10 times larger (Figure 4b). Results, that velocities of DNA in both SAMcoated nanopores were close and DNA did not stall during the time of simulation, indicate no interfacial locking in a high biasing electric field because the electric driving force is much larger than the friction force on the SAM-surface.

\section{Discussion}

We have shown experimentally and theoretically that a functionalized nanopore in a thin solid membrane, mimicking a cell membrane channel, can be used to control the transport of DNA. By switching the coated SAM in the same nanopore from being hydrophilic to being hydrophobic, we were able to change the flux and the velocity of DNA transport. MD simulations demonstrated that those changes resulted from different interaction between DNA and coated SAMs. Stronger interaction between DNA and a hydrophilic-SAMcoated nanopore slows downs the DNA transport process but can also increase the transport flux.

Modifying functional groups of a coated SAM may facilitate the development of nanopore-based DNA sequencing technologies ${ }^{22-25}$. One of the technical roadblocks that has prevented the implementation of solid-state nanopores for DNA sequencing is the absence of a reliable approach to control the translocation of DNA through the nanopore while avoiding the sticking between DNA and a pore. It has been observed that subtle properties of the inner surface of biological pores can significant affect the translocation behavior of singlestranded DNA ${ }^{26}$. Thus, with our technology to coat a desired SAM inside a nanopore, it is possible to slow down the translocation of DNA, increase the capture rate of DNA, and diminish unwanted interaction between DNA and the pore surface. The coating also enables to optimize the interaction between translocating DNA molecules and the pore surface, towards exploring the best transiting times that allow an electrical sensor to resolve the difference between four DNA nucleotides.

More generally, a SAM-coated nanopore could be used to control the transport of other biomolecules, such as proteins. In a bio-mimicking fashion, a functionalized solid-state nanopore could even be selective, becoming a sodium/potassium channel or a nutrient transporter. This may require a more complicated coating mechanism, such as differential coating or patterned coating of different types of molecules inside a nanopore drilled through a sandwich-like membrane ${ }^{27}$.

\section{Methods}

Synthesis of coating molecules. We synthesized the organic coating compound 4carboxyl benzyl phosphonic acids. (1) 4-Bromomethylbenzoic acid (2.15 g, $10 \mathrm{mM}$ ) was suspended in $10 \mathrm{~mL}$ of triethyl phosphite and the mixture was heated at $90^{\circ} \mathrm{C}$ under nitrogen for 5 hours. After cooling to room temperature, excess triethyl phosphite was removed under vacuum and the solid residue was crystallized from toluene to give diethyl 4-carboxybenzylphosphonate $(2.21 \mathrm{~g}, 81 \%)$ as white needles. NMR (DMSO-d6); ppm $1.6 \mathrm{t}, \mathrm{j}=6.2 \mathrm{~Hz}, 6 \mathrm{H} ; 3.16, \mathrm{~d}, \mathrm{j}=22 \mathrm{~Hz}, 2 \mathrm{H} ; 3.9, \mathrm{~m}, 4 \mathrm{H} ; 7.38$, $\mathrm{d},=8 \mathrm{~Hz}, 2 \mathrm{H}$ and $7.86, \mathrm{~d}, \mathrm{j}=8 \mathrm{~Hz}, 2 \mathrm{H}$. (2) Diethyl 4-carboxybenzylphosphonate (1.36 g, $5 \mathrm{mM}$ ) was suspended in $30 \mathrm{~mL}$ of $20 \%$ hydrochloric acid and the mixture was heated to reflux under nitrogen for 6 hours. After cooling to room temperature, the solvent was removed under reduced pressure and the residual solid was crystallized from isopropyl alcohol to give 4-carboxybenzylphosphonic acid (650 $\mathrm{mg}$, $61 \%)$ as white crystals, NMR (CH3OH-d4), ppm, $3.24 \mathrm{~d}, \mathrm{j}=22 \mathrm{~Hz}, 2 \mathrm{H} ; 7.43$, $\mathrm{d},=8 \mathrm{~Hz}, 2 \mathrm{H}$ and $7.97, \mathrm{~d}, \mathrm{j}=8 \mathrm{~Hz}, 2 \mathrm{H}$.

The coating of a nanopore (drilled into a $\mathrm{Si}_{3} \mathrm{~N}_{4}$ membrane) is achieved by immersing the membrane into a solution containing $2 \mathrm{mM} \mathrm{4}$ - carboxylbenzyl phosphonic acid in ethanol for 12 hours and rinsing it with ethanol afterwards for about 1 to 2 hours. The phosphonic acid group covalently and exclusively bonds to the $\mathrm{Si}_{3} \mathrm{~N}_{4}$ membrane surface and the carboxylic acid group is exposed on the SAM surface. At PH 7.0, the resulting SAM surface is neutral and hydrophilic. To switch surface functionality, a substrate coated with monolayer of 4-carboxybenzylphosphonic acid was immersed in a solution of trimethylsilyl diazomethane ( $2 \mathrm{M}$ solution in hexane, $2 \mathrm{~mL}$ ) in $50 \mathrm{~mL}$ of anhydrous tetrahydrofuran. To this solution was added $1 \mathrm{~mL}$ of methanol and kept at room temperature for 4 hours. The substrate was removed from the solution and rinsed in methanol and dried under stream of nitrogen to give methyl ester of the monolayer.

Instruments and data analyses. The platform comprises a 30 -nm-thick $\mathrm{Si}_{3} \mathrm{~N}_{4}$ membrane with a 5-nm-diameter nanopore. The membrane is mounted in a fluidic cell, separating the cis. and trans. reservoirs. The cis. reservoir is filled with an aqueous electrolyte/DNA solution. The $1 \mathrm{M} \mathrm{KCl}$ electrolyte (measured $\mathrm{PH}=6.9$, no buffer) was made using DI water (millipore, MA). The 2-K-base-pairs dsDNA was purchased from Fermentas (Thermo Scientific, PA, US). DNA-test solutions were mixed using fresh DI water and their $\mathrm{PH}$ values were confirmed by measurements, right before translocation experiments. Fluidic cells were flushed with fresh solutions before every experiment. Two $\mathrm{Ag} / \mathrm{AgCl}$ electrodes are immersed into two reservoirs, electronically connecting the flow cell to an ionic current measurement set-up. Thus, DNA molecules in the cis. compartment were electrically driven to the trans. compartment through the nanopore. The set-up includes a computer-controlled patch clamp amplifier (Axon Axopatch 200B, Molecular Devices), and a DAQ card (Digidata 1440A, Molecular Devices). All data were acquired at $250 \mathrm{KHz}$ sampling and were analyzed using a $100 \mathrm{KHz}$ low-pass filter. We extracted the mean dwell time from the histogram of dwell times by an exponential fitting.

Nanopore fabrication. The fabrication of the nanopore membrane starts from a conventional 750- $\mu$ m-thick 200-mm-diameter (100) Si wafer. Firstly, low pressure chemical vapor deposition (LPCVD) was used to deposit low-stress 30-nm-thick $\operatorname{SiN}_{x}$ on both sides of the Si wafer. Then $250 \mathrm{~nm} \mathrm{Si}{ }_{3} N_{4}$ was deposited on the backside of the Si wafer using plasma enhanced chemical vapor deposition (PECVD). 200-nm-thick PECVD $\mathrm{SiO}_{2}$ and 200-nm-thick PECVD $\mathrm{Si}_{3} \mathrm{~N}_{4}$ were deposited sequentially on the front side of the Si wafer. Then a square hole was etched through the 280 -nm-thick $\mathrm{Si}_{3} \mathrm{~N}_{4}$ (250 nm PECVD $\mathrm{Si}_{3} \mathrm{~N}_{4}$ plus $30 \mathrm{~nm} \mathrm{LPCVD} \mathrm{SiN}$ ) on the backside of the wafer into the $S i$ substrate by reactive ion etching (RIE). The $S i$ wafer was then put into $80^{\circ} \mathrm{C}, 25 \%$ (w/w) TMAH solution to etch away the backside $\mathrm{Si}$ material. The etching was anisotropic, with the etch rate in (100) direction being much larger than the one in (111) direction. Hence, an inverted pyramid is etched into Si material before the etch stops on the 30-nm-thick LPCVD $\mathrm{Si}_{3} \mathrm{~N}_{4}$, forming a $100 \mu \mathrm{m} \times 100 \mu \mathrm{m}$ squareshaped free-standing membrane made of $30-\mathrm{nm}-\mathrm{Si}_{3} \mathrm{~N}_{4} / 200-\mathrm{nm}-\mathrm{SiO}_{2} / 200-\mathrm{nm}-\mathrm{Si}_{3} \mathrm{~N}_{4}$ (from bottom to top). A 5- $\mu$ m-diameter hole was made at the center of the freestanding membrane, by etching through the top two layers $\left(200 \mathrm{Si}_{3} \mathrm{~N}_{4} / 200 \mathrm{~nm} \mathrm{SiO}\right.$ ) and creating a free-standing membrane area (made of 30-nm-thick SiN). A nanopore (typical diameters ranging from $3 \mathrm{~nm}$ to $10 \mathrm{~nm}$ ) was then drilled through the freestanding membrane using a focused electron beam in a transmission electron microscope (JEOL 3000F). 
Molecular dynamics simulation. All-atom molecular dynamics (MD) simulations were carried out on the IBM Bluegene supercomputer, using the software package $\mathrm{NAMD}^{28}$. The CHARMM27 force-field ${ }^{29}$ was used to model DNA and organosilane molecules. We applied the TIP3P model $^{30}$ for water molecules, silica force field ${ }^{31}$ for a $\mathrm{SiO}_{2}$ solid, and standard force fields ${ }^{32}$ for ions. All simulations were carried out in the NVT $(T=300 \mathrm{~K})$ ensemble. To maintain the system at constant temperature $T$, Langevin dynamics was applied to atoms in the $\mathrm{SiO}_{2}$ solid. These atoms were additionally restrained harmonically to occupy amorphous lattice sites. Each simulation system measures $96 \times 96 \times 32 \AA^{3}$. With periodic boundary conditions in all three dimensions, long-range coulomb interactions were computed using particlemesh Ewald (PME) full electrostatics. The van der Waals interaction between atoms was calculated using a smooth (10-12 $\AA$ ) cutoff.

1. Preston, G. M., Carroll, T. P., Guggino, W. B. \& Agre, P. Appearance of water channels in xenopus oocytes expressing red cell chip28 protein. Science 256, 385-387 (1992).

2. Kasianowicz, J. J. Introduction to ion channels and disease. Chemical Reviews $\mathbf{1 1 2}$ 6215-6217 (2012)

3. Dekker, C. Solid-state nanopores. Nature Nanotech. 2, 209-215 (2007).

4. Venkatesan, B. \& Bashir, R. Nanopore sensors for nucleic acid analysis. Nature Nanotech. 6, 615-624 (2011)

5. Cressiot, B. et al. Protein transport through a narrow solid-state nanopore at high voltage: experiments and theory. ACS nano 6, 6236-6243 (2012).

6. Zhao, Q. et al. Detecting snps using a synthetic nanopore. Nano Lett. 7, 1680-1685 (2007)

7. Hall, A., van Dorp, S., Lemay, S. \& Dekker, C. Electrophoretic force on a proteincoated dna molecule in a solid-state nanopore. Nano letters $9,4441-4445$ (2009).

8. Li, J. et al. Ion-beam sculpting at nanometre length scales. Nature 412, 166-169 (2001).

9. Wanunu, M., Sutin, J., McNally, B., Chow, A. \& Meller, A. DNA translocation governed by interactions with solid-state nanopores. Biophys. J. 95, 4716-4725 (2008).

10. Yusko, E. et al. Controlling protein translocation through nanopores with bioinspired fluid walls. Nature Nanotech. 6, 253-260 (2011).

11. Iqbal, S., Akin, D. \& Bashir, R. Solid-state nanopore channels with DNA selectivity. Nature Nanotech. 2, 243-248 (2007).

12. Hall, A. et al. Hybrid pore formation by directed insertion of $\alpha$-haemolysin into solid-state nanopores. Nature Nanotech. 5, 874-877 (2010).

13. Bell, N. A. et al. DNA origami nanopores. Nano Lett. 12, 512-517 (2012).

14. Wanunu, M. \& Meller, A. Chemically modified solid-state nanopores. Nano Lett. 7, 1580-1585 (2007)

15. Charles, R. M. \& Zuzanna, S. S. Learning nature's way: Biosensing with synthetic nanopores. Science 317, 331 (2007)

16. Wei, R., Gatterdam, V., Wieneke, R., Tampé, R. \& Rant, U. Stochastic sensing of proteins with receptor-modified solid-state nanopores. Nature Nanotech. 7, 257-263 (2012).

17. Berezhkovskii, A. M. \& Bezrukov, S. M. Optimizing transport of metabolites through large channels: Molecular sieves with and without binding. Biophys. J. 88 L17-L19 (2004)

18. Smeets, R. M. M. et al. Salt dependence of ion transport and DNA translocation through solid-state nanopores. Nano Lett. 6, 89-95 (2006).

19. Wanunu, M., Morrison, W., Rabin, Y., Grosberg, A. Y. \& Meller, A. Electrostatic focusing of unlabelled DNA into nanoscale pores using a salt gradient. Nature Nanotech. 5, 160-165 (2010).

20. Luan, B. et al. Base-by-base ratcheting of single stranded DNA through a solid state nanopore. Phys. Rev. Lett. 104, 238103 (2010).

21. Luan, B. Q., Martyna, G. \& Stolovitzky, G. Characterizing and controlling the motion of ssDNA in a solid-state nanopore. Biophys. J. 101, 2214 (2011).
22. Kasianowicz, J. J., Brandin, E., Branton, D. \& Deamer, D. W. Characterization of individual polynucleotide molecules using a membrane channel. Proc. Natl. Acad. Sci. USA 93, 13770-13773 (1996).

23. Branton, D. et al. The potential and challenges of nanopore sequencing. Nature Biotech. 26, 1146-1153 (2008).

24. Xie, P., Xiong, Q., Fang, Y., Qing, Q. \& Lieber, C. Local electrical potential detection of DNA by nanowire-nanopore sensors. Nature Nanotechnology 7, 119-125 (2012)

25. Ivanov, A. et al. DNA tunneling detector embedded in a nanopore. Nano Lett. 11, 279 (2011).

26. Rincon-Restrepo, M., Mikhailova, E., Bayley, H. \& Maglia, G. Controlled translocation of individual DNA molecules through protein nanopores with engineered molecular brakes. Nano Lett. 11, 746-750 (2011).

27. Polonsky, S., Rossnagel, S. \& Stolovitzky, G. Nanopore in metal-dielectric sandwich for DNA position control. Appl. Phys. Lett. 91, 153103 (2007).

28. Phillips, J. C. et al. Scalable molecular dynamics with NAMD. J. Comp. Chem. 26 1781-1802 (2005).

29. MacKerell, A., Jr. et al. All-atom empirical potential for molecular modeling and dynamics studies of proteins. J. Phys. Chem. B 102, 3586-3616 (1998).

30. Jorgensen, W. L., Chandrasekhar, J., Madura, J. D., Impey, R. W. \& Klein, M. L. Comparison of simple potential functions for simulating liquid water. J. Chem. Phys. 79, 926-935 (1983).

31. Cruz-Chu, E. R., Aksimentiev, A. \& Schulten, K. Water-silica force field for simulating nanodevices. J. Phys. Chem. B 110, 21497-21508 (2006).

32. Beglov, D. \& Roux, B. Finite representation of an infinite bulk system: Solvent boundary potential for computer simulations. J. Chem. Phys. 100, 9050-9063 (1994).

\section{Acknowledgments}

The authors gratefully acknowledge the microelectronics Research Laboratory (MRL) and specifically Phil Waggoner and Stephen Rossnagel at the IBM T. J. Watson Research Center for assistance with fabricating membrane devices. We express our gratitude towards Kathy Reuter for pore drilling and imaging support, and Stanislav Polonsky as well as Young Kwark for their help in calibrating the electronic measurement setup. Ajay Royyuru provided valuable advice. The work presented was supported by a grant (Number: R01HG005110) from the National Human Genome Research Institute.

\section{Author contributions}

All discussed and designed the project. D.W. and S.H. performed experiments of nanopore coating and DNA translocation. B.L. did MD simulations. H.P. fabricated the $\mathrm{SiNx}$ membranes. A.A. synthesized the coating molecules. D.W., S.H. and B.L. produced all the figures. B.L., D.W., G.S. and S.H. wrote the paper.

\section{Additional information}

Supplementary information accompanies this paper at http://www.nature.com/ scientificreports

Competing financial interests: The authors declare no competing financial interests.

How to cite this article: Wang, D.Q. et al. Regulating the Transport of DNA through Biofriendly Nanochannels in a Thin Solid Membrane. Sci. Rep. 4, 3985; DOI:10.1038/ srep03985 (2014).

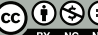

This work is licensed under a Creative Commons AttributionNonCommercial-NoDerivs 3.0 Unported license. To view a copy of this license, visit http://creativecommons.org/licenses/by-nc-nd/3.0 